\title{
Study on Capturing PM2.5 Capability of Tree Species in Different Functional Areas
}

\author{
J. Yang \& B.Z. Xie \& H. Shi \\ School of Environmental and Municipal Engineering, Xi'an University of Architecture and Technology, Xi'an \\ 710055, China
}

\author{
H.X. Wang \& Y.H. Wang \\ Institute of Forest Ecology, Environment and Protection, Chinese Academy of Forestry, Beijing 100091, \\ China
}

\begin{abstract}
Trees can capture PM on account of their foliage's structure, thus, improving air quality. We determined leaf PM-retaining capability $\left(\mathrm{PM}_{2.5}, \mathrm{PM}_{>2.5}, \mathrm{PM}\right)$ of evergreen shrub (Euonymus japonicas) and decicuous trees (Sophora japonica, Fraxinus chinensis, Ginkgo biloba, Populus tomentosa) in different functional areas. The leaf micromorphological structure was observed using a field emission environmental scanning electron microscopy. We found that E. japonicas should be planted in traffic and industrial areas, while S. japonica, G. biloba, F. chinensis should be chosen as complementary in clean areas. S. japonica, G. biloba were adapted well to polluted environment, but, F. chinensis was not.
\end{abstract}

KEYWORD: CAPTURING PM 2.5 ; DIFFERENT FUNCTIONAL AREAS; structural feature

\section{INTRODUCTION}

It has been shown that particulate matter (PM) causes greater damage with smaller diameter (Pope et al. 2002). PM2.5 (particles with diameter less than $2.5 \mu \mathrm{m}$ ), comprising a lot of poisonous and harmful substances, can deposit in alveolus through inhalation. And $\mathrm{PM}_{2.5}$ can stay for a long time in the atmosphere and transfer widely. Therefore, it can produce great harm to human health and atmospheric environment quality (Zhang et al. 2002). Trees can capture PM due to their foliage's structure to improve air quality (Nowak et al. 2006).

In recent years, scholars have gotten many results on trees' capability of capturing PM at home and abroad (Liu et al. 2013; Räsänen et al. 2013). Wang et al. (2012) found that the amount, distribution, and morphology of trichomes had great influence on the leaf PM-retaining capability. Using oscillating mode AFM (atomic force microscopy), Shi et al. (2011) found that many papillae and hollows on the adaxial surface of Ligustrum lucidum and Viburnum odoratissimum with a radius of about $10 \mu \mathrm{m}$ can capture $\mathrm{PM}_{10}$ (particles with diameter less than 10 $\mu \mathrm{m})$. Existing research mainly focus on PM and $\mathrm{PM}_{10}$ or only a particular functional areas. Few results are available on trees stranding $\mathbf{P M}_{2.5}$ in different functional areas.

This paper selected three different functional areas: Beijing Botanical Garden (clean areas), Guomao Bridge (traffic areas), Huangcun (industrial areas), as researching areas. We measured and compared the amount of capturing $\mathrm{PM}_{>2.5}, \mathrm{PM}_{2.5}$, and PM per unit leaf area of five common greening tree species (E. japonicas, $S$. japonica, $F$. chinensis, $G$. biloba, $P$. tomentosa), and observed the leaf micromorphological structure with a scanning electron microscopy.

\section{MATERIALS AND METHODS}

\subsection{Sampling site}

Beijing is located in $39^{\circ} 26^{\prime}-41^{\circ} 03^{\prime} \mathrm{N}, 115^{\circ} 25^{\prime}-$ $117^{\circ} 30^{\prime} \mathrm{E}$, with average annual precipitation $644 \mathrm{~mm}$, and the precipitation from June to August accounts for about $80 \%$ of the whole year. Sampling sites are Beijing Botanical Garden (clean areas), Guomao Bridge (traffic areas), Huangcun (industrial areas).

\subsection{Methods}

\subsubsection{Plant materials and Sampling procedure}

Five common greening trees (E. japonicas, $S$. japonica, F. chinensis, G. biloba, P. tomentosa) were selected for analysis in the three functional areas. E. japonicas is the largest amount of evergreen shrubs in Beijing, and S. japonica, F. chinensis, $G$. biloba, $P$. tomentosa account for over $60 \%$ of deciduous trees in Beijing. All leaf sampling was conducted on October 1 in 2013 in order to reduce the error caused by different sampling time, without rainfall for more than a week. Sampling height of 
deciduous trees was about $2.5 \mathrm{~m}$, and that of evergreen shrub was about $1.0 \mathrm{~m}$. All sample trees were not suffering from obvious pests or diseases. During the experiment, samples were stored at $4^{\circ} \mathrm{C}$ in fridge.

\subsubsection{Analysis of PM}

For each tree species, three batches of test material were prepared. And for each batch, 50-60 leaves were selected. The leaves were washed with a brush. The total hemi-surface leaf area was measured using Image J software (Version 1.46; National Institutes of Health, USA) after scanned (HP Scanjet 3570c, Japan). As for the filtration procedure, we used filter membranes with the pore of $2.5 \mu \mathrm{m}$, these filter membranes were pre-weighed after $24 \mathrm{~h}$ drying at $40^{\circ} \mathrm{C}$ using a balance with $0.1 \mathrm{mg}$ accuracy (SI-114, Denver Instrument, USA). Particles in filter membranes was $\mathrm{PM}_{>2.5}$ (particles with diameter greater than $2.5 \mu \mathrm{m}$ ). In addition, filtered filtrate were dried to constant weight with baker immediately, thus, only $\mathrm{PM}_{2.5}$ was left. The resulting weight was finally divided by leaf area. We obtained the weight of deposited $\mathrm{PM}_{>2.5}$ and $\mathrm{PM}_{2.5}$ per unit leaf area for each washed leaf sample. PM was the sum of $\mathrm{PM}_{>2.5}$ and $\mathrm{PM}_{2.5}$.

\subsubsection{Analysis of leaf surfaces characteristics}

For each of the specie, three leaves were randomly selected, and cut into $5 \mathrm{~mm} \times 5 \mathrm{~mm}$ samples from different parts of the leaf avoiding main vein. Then the samples were adhered to the aluminum tube using double-sided adhesive tape. The field emission scanning electron microscope (FESEM, Quanta 200,
FEG, USA) was used to observe the upper and lower leaf surfaces characteristics.

\subsubsection{Data analysis}

One-way analysis of variance (ANOVA) was conducted to test the differences in $\mathrm{PM}, \mathrm{PM}_{>2.5}$, $\mathrm{PM}_{2.5}$ at each sampling sites. When there was significant difference, the Student-Newman-Keuls multiple comparison was performed to analyze for each pair. All the analysis were performed using SPSS 20 (IBM, USA). A given effect was assumed as significant at $p=0.05$.

\section{RESULTS}

\subsection{Differences among species}

The amount of $\mathrm{PM}, \mathrm{PM}_{>2.5}$ deposited on leaves was significantly different among tree species $(p<0.01$, Table 1). Accumulated PM of E. japonicas was the biggest in three functional areas; in traffic and industrial areas, $S$. japonica followed, and $P$. tomentosa was the smallest; but in clean areas the smallest was $S$. japonica. The average accumulated $\mathrm{PM}_{>2.5}$ of five tree species in three functional areas were $94 \%, 91 \%$ and $92 \%$ of PM, thus, accumulated $\mathrm{PM}_{>2.5}$ of tree species' order was the same as PM.

The amount of $\mathrm{PM}_{2.5}$ deposited on leaves was significantly different among tree species in traffic areas and industrial areas $(p<0.05$, Table 1$)$, but, there was not significantly different in clean areas $(p>0.05$, Table 1). In clean and industrial areas, accumulated $\mathrm{PM}_{2.5}$ of $S$. japonica was the biggest, and it was only less than E. japonicas in traffic; $P$. tomentosa was the smallest in three functional areas.

Table 1 Particle size fractions captured $\left(\mathrm{g} / \mathrm{m}^{2}\right)$ on unit leaf area of trees in different functional areas

\begin{tabular}{|l|c|c|c|c|c|c|c|c|c|}
\hline & \multicolumn{3}{|l|}{ Beijing Botanical Garden } & \multicolumn{3}{l|}{ Guomao Bridge } & \multicolumn{3}{l|}{ Huangcun } \\
\hline & $\mathrm{PM}_{2.5}$ & $\mathrm{PM}_{>2.5}$ & $\mathrm{PM}$ & $\mathrm{PM}_{2.5}$ & $\mathrm{PM}_{>2.5}$ & $\mathrm{PM}$ & $\mathrm{PM}_{2.5}$ & $\mathrm{PM}_{>2.5}$ & $\mathrm{PM}$ \\
\hline Euonymus japonicas & 0.05 & 2.10 & 2.15 & 0.43 & 5.74 & 6.17 & 0.24 & 5.86 & 6.10 \\
\hline Sophora japonica & 0.08 & 0.58 & 0.66 & 0.16 & 1.25 & 1.41 & 0.29 & 1.77 & 2.06 \\
\hline Fraxinus chinensis & 0.07 & 1.04 & 1.11 & 0.10 & 0.72 & 0.82 & 0.12 & 1.14 & 1.26 \\
\hline Ginkgo biloba & 0.06 & 0.92 & 0.98 & 0.09 & 1.16 & 1.25 & 0.11 & 1.31 & 1.42 \\
\hline Populus tomentosa & 0.04 & 0.86 & 0.90 & 0.05 & 0.71 & 0.76 & 0.06 & 1.04 & 1.10 \\
\hline
\end{tabular}

\subsection{Differences in $P M, P M_{>2.5}$ and $P M_{2.5}$ among three functional areas}

The average $\mathrm{PM}, \mathrm{PM}_{>2.5}, \mathrm{PM}_{2.5}$ per unit leaf area in traffic and industrial areas were significantly higher than those in clean areas, by 1.80 and $2.05,1.75$ and 2.03, 2.83 and 2.67 folds.

In traffic areas, the ratio of accumulated PM of $E$. japonicas, S. japonica, G. biloba to those of clean areas was $2.87,2.14,1.40$, but $F$. chinensis, $P$. tomentosa were less than those of clean areas, and had only their 74\%, 84\%; however, in industrial areas, E. japonicas, S. japonica, G. biloba, $F$. chinensis, $P$. tomentosa was $2.84,3.12,1.45,1.14$ and 1.22. In addition, in industrial areas, the amount of $\mathrm{PM}_{>2.5}$ of five tree species were larger than that of clean areas, $F$. chinensis, $P$. tomentosa in traffic areas were only less than those of clean areas.

Capturing $\mathrm{PM}_{2.5}$ of E. japonicas, S. japonica, $G$. biloba, $F$. chinensis, $P$. tomentosa in traffic and 
industrial areas were larger than those of clean areas, by 8.60 and $4.80,2.00$ and $3.63,1.50$ and $1.83,1.43$ and $1.71,1.25$ and 1.50 folds (Table 1 ).

\subsection{Morphological structure of leaf surfaces and changes caused by environment}

With FESEM, it was found that morphological structure of leaf surfaces of E. japonicas, lower leaf surfaces of $S$. japonica, lower leaf surfaces of $G$. biloba, and leaf surfaces of $P$. tomentosa without significant changing (Table 2). However, structure of leaf surfaces of others tree species change greatly. In traffic areas, upper leaf surfaces of E. japonicas had more trichomes, massive grooves and protuberances than those of clean areas, but, in industrial areas, leaf surfaces were packaged by thick wax. In addition, the upper leaf surfaces of $F$. chinensis in industrial areas had higher bar protuberances compared to that in clean areas, however, there was not in traffic areas. The upper leaf surfaces of $F$. chinensis had only trichomes in clean areas. And the grooves' depth of upper leaf surfaces of G. biloba: industrial areas $>$ traffic areas > clean areas (figure 1).

Table 2 Structure characteristics of leaf surfaces of tree species

\begin{tabular}{|l|l|}
\hline & structural feature of leaf surfaces \\
\hline upper leaf surfaces of Euonymus japonicas & smooth leaf surfaces and low protuberances \\
\hline lower leaf surfaces of Euonymus japonicas & massive protuberances \\
\hline lower leaf surfaces of Sophora japonica & trichomes and massive shallow grooves \\
\hline lower leaf surfaces of Ginkgo biloba & bits of protuberances \\
\hline upper leaf surfaces of Populus tomentosa & smooth leaf surfaces and lower grooves \\
\hline lower leaf surfaces of Populus tomentosa & bits of banded structures \\
\hline
\end{tabular}
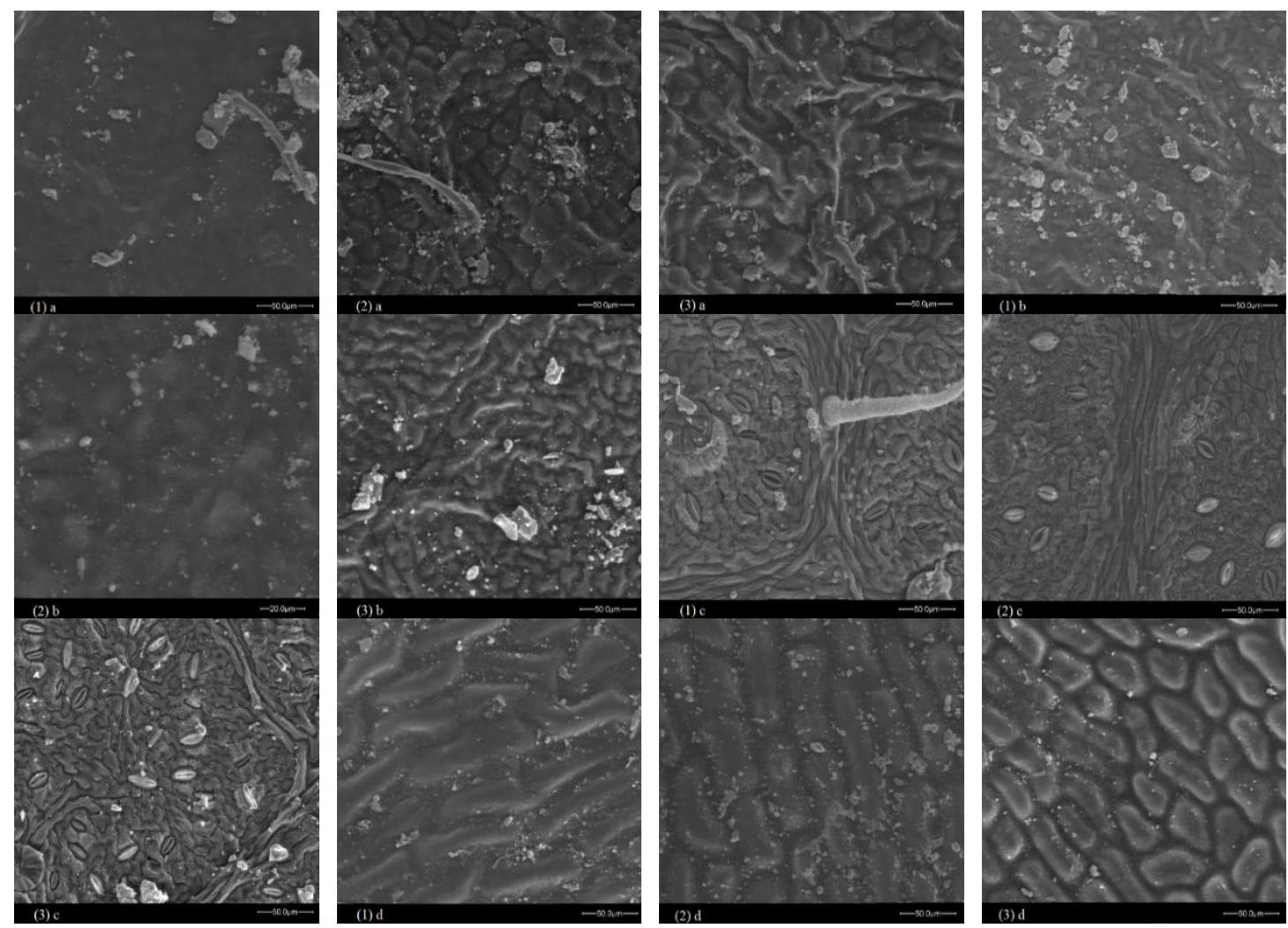

Fig.1 Scanning electron microscope pictures. Upper leaf surfaces of Sophora japonica (a), upper leaf surfaces of Fraxinus chinensis (b), lower leaf surfaces of Fraxinus chinensis (c), upper leaf surfaces of Ginkgo biloba (d). Clean areas (1), traffic areas (2), industrial areas (3).

\section{DISCUSSION}

Difference of PM-retaining capability among tree species was determined by leaf morphological structure (Chai et al. 2002), and leaves with bigger roughness and more trchiomes were advantage to capture PM (Freer et al. 1997; Wang et al. 2012). Liang et al. (2005) found that the PM-retaining per unit area of shrubs were larger than of evergreen trees and deciduous trees. In traffic and industrial 
areas, the amount of accumulated $\mathrm{PM}, \mathrm{PM}_{>2.5}, \mathrm{PM}_{2.5}$ of evergreen shrub E. japonicas was the biggest, because its height is close to breathing area of the human body. With scanning electron microscope, it was found particles covered the leaf surfaces densely, but their growth was still good. In clean areas, the amount of accumulated $\mathrm{PM}_{2.5}$ of $E$. japonicas was less than of $S$. japonica, G. biloba, $F$. chinensis. The reason was that there was less fine particles in atmosphere and leaf surfaces of $S$. japonica, G. biloba, F. chinensis were more rough than E. japonicas. Thus, in traffic and industrial areas, we should plant E. japonicas, and in clean areas $S$. japonica, G. biloba, F. chinensis should be chosen as complementary.

In traffic areas, the leaf surfaces of $S$. japonica had massive grooves and protuberances. It captured less $\mathrm{PM}, \mathrm{PM}_{>2.5}, \mathrm{PM}_{2.5}$ than evergreen shrub $E$. japonicas did. In addition, surfaces of leaves of $S$. japonica were covered by wax, leading to a small affinity between particles and leaf surface (Koch et al. 2009). The upper leaf surfaces of $F$. chinensis had massive deeper grooves in industrial areas to improve PM-retaining capability. Wang et al. (2006) had shown that the capability of dust-retaining was higher when the micro-configurations were denser. Changing of grooves' depth of upper leaf surfaces of G. biloba improved their PM-retaining capability. Thus, S. japonica, G. biloba were adapted well to polluted environment, but, $F$. chinensis was not.

\section{REFERENCES}

[1] Pope III C.A. \& Burnett R.T. \& Thun M.J. \& Calle E.E. \& Krewski D. \& Ito K. \& Thurston G.D. 2002. Lung cancer, cardiopulmonary mortality, and long-term exposure to fine particulate air pollution. Journal of the American Medical Association, 287(9):1132-1141.

[2] Zhang W.L. \& Xu D.Q. \& Cui J.S. 2002. The characteristics and toxic mechanism of fine particle pollution $\left(\mathrm{PM}_{2.5}\right)$ in air. Environmental Monitoring in China, 18(1):59-63.

[3] Nowak D.J. \& Crane D.E. \& Steven J.C. 2006. Air pollution removal by urban trees and shrubs in the United States. Urban Forestry \& Urban Green, 4(3-4):115-123.

[4] Liu L. \& Guan D.S. \& Chen Y.Q. 2013. Morphological structure of leaves and dust-retaining capability of common street trees in Guangzhou Municipality. Acta Ecologica Sinica, 33(8):2604-2614.

[5] Räsänen J.V. \& Holopainen T. \& Joutsensaari J. \& Ndam C. \& Pasanen P. \& Rinnan A. \& Kivimäenpää M. 2013. Effects of species-specific leaf characteristics and reduced water availability on fine particle capture efficiency of trees. Environmental Pollution, 183:64-70.

[6] Wang H.X. \& Shi H. \& Li Y.Y. 2012. Relationships between leaf surface characteristics and dust-capturing capability of urban greening plant species. Chinese Journal of Applied Ecology, 21(12):3077-3082.

[7] Shi H. \& Wang H.X. \& Li Y.Y. \& Liu X. 2011. Leaf surface microstructure of Ligustrum lucidum and Viburnum odoratissimum observed by Atomic force microscopy (AFM). Acta Ecologica Sinica, 31(5):1471-1477.

[8] Chai Y.X. \& Zhu N. \& Han H.J. 2002. Dust removal effect of urban tree species in Harbin. Chinese Journal of Applied Ecology, 13(9):1121-1126.

[9] Freer Smith P.H. \& Holloway S. \& Goodman A. 1997. The uptake of particulates by an urban woodland: Site description and particulate composition. Environment Pollution, 95(1):27-35.

[10]Kouch K. \& Bhushan B. \& Barthlott W. 2009. Multifunctional surface structures of plants: An inspiration for biomimetics. Progress in Materials Science, 54:137178.

[11]Wang L. \& Gao S.Y. \& Liu L.Y. \& Ha S. 2006. Atmospheric particle-retaining capability of eleven garden plant species in Beijing. Chinese Journal of Applied Ecology, 17(4):597-61. 